\title{
Electron Tomography of Hydrated Ferritin Particles Using Carbon Nanotube Liquid Cell
}

\author{
C. Wang ${ }^{1}$, S. Sinha-Ray ${ }^{2}$, A.L. Yarin ${ }^{2}$, T. Shokuhfar ${ }^{1,3}$, R.F. Klie ${ }^{1}$ \\ ${ }^{1}$ Department of Physics, University of Illinois at Chicago, Chicago, IL 60607 \\ ${ }^{2}$ Department of Mechanical and Industrial Engineering, University of Illinois at Chicago, IL 60607 \\ ${ }^{3}$.Department of Mechanical Engineering, Michigan Technological University, Houghton, MI, 49931
}

Cryo-electron microscopy tomography has been extensively used in modern biological research to study the constituents and structures of cells and other biological samples. In cryo-electron microscopy, the cellular structures are preserved by converting cellular water into amorphous ice via the preparation of cryo sections [1,2], since most electron microscopes are incompatible with hydrated samples. However, because of this the observed cells are no longer in their native liquid state, subject to possibility of change of the structure, and resulting in inability to examine details of dynamic processes. Therefore, to study the dynamic process, as well as the structures of unperturbed biological systems, there is a pressing need for electron tomography of cells and other biological samples in the liquid state at room temperature. However, conventional liquid stages currently available are not designed to do high resolution electron tomography.

In this presentation we introduce a bio-compatible carbon nanotube based liquid cell which can be used on a conventional electron microscope tomography stage. Following the novel diffusion driven intercalation process [3-5], ferritin liquid containing ferritin protein was intercalated within carbon nanotubes. Initially, the carbon nanotube powder was mixed with sericin in the ferritin solution in a beaker. The beaker is then placed inside a desiccator, allowing the vapor of the mixed solution to be constantly pumped out through a vacuum line. The temperature of the desiccator was kept between $2 \sim 8{ }^{\circ} \mathrm{C}$, to preserve the biological activity of the ferritin particle. This ensured concentration gradient [3-5] resulting in fill up of ferritin inside CNTs. After 48 hours, the carbon nanotube was examined in the electron microscope.

High-angle annular dark-field images show the sericin (a hydrogel), soaked in water and forming meniscus inside the carbon nanotube (Figure 1), as well as the distribution of the ferritin particles inside the carbon nanotube (Figure 2). Electron tomography was used to confirm the encapsulation of ferritin particles inside the carbon nanotube. In the reconstruction model, ferritin particles were shown to adhere to the inner wall of the carbon nanotube.

Utilizing a carbon nanotube liquid cell as described above, we will reconstruct the 3-dimentional structure of the ferritin particles in their hydrated condition using the aberration-corrected JEOL ARM200-CF operated at $80 \mathrm{kV}$, and show that the bio-nano interface of ferritin in a hydrated condition can be resolved using this method. This provides a method to perform tomography on biological samples in their parent hydrated condition, which the present state of the art methods can't provide. The CNT liquid cell can also be used as a drug or bio-sample delivery container to preserve the drug activity or biological activity before reaching the desired destination in the body [6]. Moreover, atomic-resolution TEM/STEM images of bio-sample in a liquid environment can potentially be obtained using this method by reducing the wall thickness of the carbon nanotube. [7] 
References:

[1] H. Stahlberg, T. Walz, ACS chemical biology 3, 268-281 (2008).

[2] J. Pierson, M. Sani, C. Tomova, S. Godsave, P. J. Peters Histochemistry and cell biology 132(3), 253-262 (2009).

[3] A.V. Bazilevsky, K. Sun, A.L. Yarin, C.M. Megaridis. J. Materials Chem. 18, 696 - 702 (2008).

[4] A.V. Bazilevsky, K. Sun, A.L. Yarin, C.M. Megaridis. Langmuir, 23, 7451-7455 (2007).

[5] S. Sinha Ray, R.P. Sahu, A.L. Yarin. Soft Matter, 7, 8823-8827 (2011).

[6] Kam, N. W. Shi, Z. Liu, and H. Dai. Journal of the American Chemical Society 127.36 (2005): 12492-12493.

[7] This research was supported by Michigan Technological University and Research Resource Center, University of Illinois at Chicago.

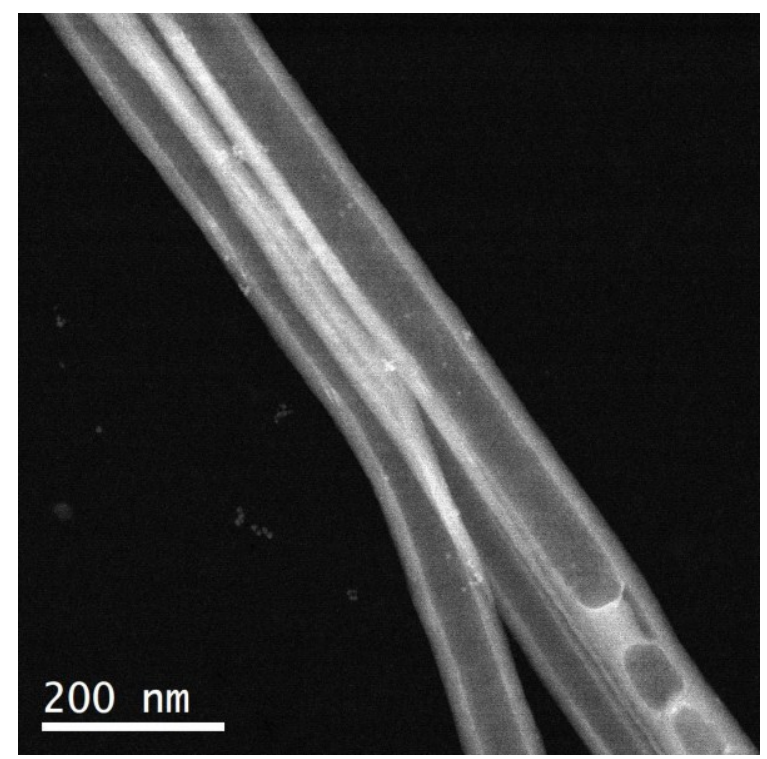

Figure 1. HAADF image showing the sericin sponge formed inside the CNT

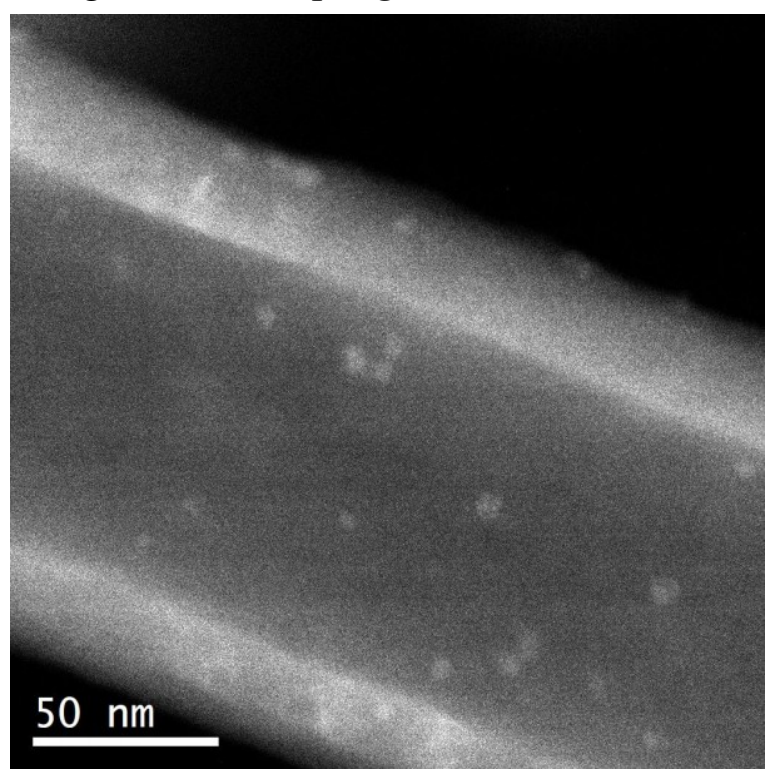

Figure 2. HAADF image showing distribution of ferritin particles inside the CNT 